\title{
A HUGHES-LIKE PROPERTY FOR FINITE GROUPS
}

\author{
by R. A. BRYCE, V. FEDRI and L. SERENA
}

(Received 30th May 1994)

\begin{abstract}
Several structure theorems are proved for groups $G$ having the following property. There is a prime $p$ and a collection of subgroups of $G$ such that the elements of $G$ which lie in the complement of every subgroup of the collection all have order $p$.
\end{abstract}

1991 Mathematics Subject Classification: 20E34.

\section{Introduction}

Let $p$ be a prime, $G$ a finite group and $\mathscr{A}$ a union of subgroups of $G$. We say that $\mathscr{A}$ has the Hughes property for exponent $p$ if the following two conditions hold: firstly $G \neq \mathscr{A}$, and secondly every element of $G \backslash \mathscr{A}$ has order $p$. (The term 'union' is used here in the sense of set theory: $\mathscr{A}$ need not be a subgroup of $G$.) The following well-known result describes the structure of a finite group which has a subgroup with the Hughes property.

Theorem 1.1. (Hughes and Thompson [2], Kegel [4]). Let $p$ be a prime, let $G$ be a finite group and let $H$ be a subgroup of $G$ with the Hughes property for exponent $p$. Then $H$ is nilpotent and, if $G$ is not a p-group, the index of $H$ in $G$ is $p$.

A more familiar statement of this is that in a non-nilpotent finite group the Hughes subgroup, that generated by the elements whose order is not $p$, if not the whole group, is nilpotent and of index $p$ in $G$.

We will denote by $\mathscr{H}_{n}(p)$ the class of all finite groups which have a union of $n$ subgroups with the Hughes property for exponent $p$. Theorem 1.1 says, among other things, that the groups of the class $\mathscr{H}_{1}(p)$ have a nilpotent normal $p$-complement. The aim of this article is to prove results like this about more general classes $\mathscr{H}_{n}(p)$.

Theorem 1.2. Let $n$ be a positive integer and $p$ a prime greater than $n$. Each group in the class $\mathscr{H}_{n}(p)$ has a nilpotent normal p-complement.

Theorem 1.3. Let $G$ be a group in the class $\mathscr{H}_{n}(p)$, and let $\pi$ be the set of primes other than $p$, which are greater than or equal to $n$. Then $O_{\pi}(G)$ is nilpotent and $G / O_{\pi}(G)$ is a $\pi^{\prime}$-group.

It is of interest to examine what these theorems say about $\mathscr{H}_{n}(p)$-groups when $n$ is small. When $n=1$ Theorem 1.2 follows from Theorem 1.1. When $n=2$ all odd primes 
are covered by Theorem 1.2. In fact the restriction to odd $p$ here is not necessary: the article [1], where techniques quite different to those in this paper are employed, proves Theorem 1.2 for $n=2$ and all $p$. For $n=3$ all primes $p$ greater than or equal to 5 are covered by Theorem 1.2. The class $\mathscr{H}_{3}(3)$ does not come under Theorem 1.2. Indeed an $\mathscr{H}_{3}(3)$-group need not be 3-nilpotent, witness the symmetric group on three symbols: the union of its three Sylow 2-subgroups has complement consisting only of elements of order 3. However we can use Theorems 1.2 and 1.3 to prove the following result.

Corollary 1.4. Let $n$ be a positive integer and $p$ a prime such that $(n, p)<(5,5)$ in the lexicographical ordering of ordered pairs. Then the groups in the class $\mathscr{H}_{n}(p)$ are soluble.

Proof. By Theorem 1.2 we may suppose that $p \leq n$. Let $\pi$ be the set of primes greater than or equal to $n$ and different from $p$. Then $\left|\pi^{\prime}\right| \leq 2$, so the result follows from Theorem 1.2 and Burnside's Theorem.

This result is best possible in the sense that $\mathscr{H}_{5}(5)$ contains the insoluble group $A_{5}$, the alternating group on five symbols: there is in $A_{5}$ a conjugacy class of five copies of $A_{4}$ whose union excludes only elements of order 5 .

More detailed structure of the groups in classes $\mathscr{H}_{n}(p)$ will be developed in a sequel to the present article.

\section{Preliminary results and notations}

If, in the sequel, the number $n$ of subgroups forming a union is important, we call it an n-union. We do not suppose in this definition that the union is necessarily irredundant, and certainly a union may be a union of subgroups in different ways. In particular we note that $\mathscr{H}_{n-1}(p) \subseteq \mathscr{H}_{n}(p)(n>1)$.

Let $G$ be a group and $\mathscr{A}$ an $n$-union with the Hughes property of sub-groups of $G$. If $H$ is a subgroup of $G$, not contained in $\mathscr{A}$, then $H \cap \mathscr{A}$ is an m-union of subgroups of $H$ with the Hughes property, for some $m \leq n$. It will sometimes be useful to write $\cap \mathscr{A}$ for the intersection of some particular expression of $\mathscr{A}$ as a union, recognising that it is not a well-defined notation.

We begin with a simple, but very useful, lemma.

Lemma 2.1. Let $G$ be a group and $\mathscr{A}$ a union with the Hughes property for exponent p. Then $\mathbf{C}_{G}(x)$ is a p-group for every $x \notin \mathscr{A}$.

Proof. If a $p^{\prime}$-element $y$ of $G$ centralises $x$, then $x y$ is not of order $p$, so lies in $\mathscr{A}$ and therefore $x$ is in $\mathscr{A}$, contradiction.

The following lemma will also be very useful to us.

Lemma 2.2. Suppose that a group $G$ is irredundantly the union of $n$ sub-groups whose intersection is D. Every p-element of $G$ is in $D$ whenever $p$ is a prime greater than or equal to $n$.

Proof. Suppose that 


$$
G=A_{1} \cup A_{2} \cup \ldots \cup A_{n}
$$

irredundantly.

Let $S$ be a non-empty subset of the set $Z=\{1,2, \ldots, n\}$ of the first $n$ positive integers. Write

$$
A_{S}=\cap\left(A_{i}: i \in S\right)
$$

For $i \in \mathbf{Z}$ we will write $A_{i}$ instead of $A_{\{i\}}$.

If $S$ is a proper subset of $Z$ we show that, for some non-empty subset $T$ of the complement of $S$ in $Z$, and for some elements $g_{t}(t \in T)$ of $G$,

$$
A_{S}=\cup\left(g_{t} A_{S \cup\{t\}}: t \in T\right)
$$

For, let $u \in \mathbf{Z} \backslash S$. If $A_{S}=A_{S \cup\{u\}}$ then the claim is immediate: simply choose $T=\{u\}$. If not, let $a$ be an element of $A_{u}$ which is not in any of the other $A_{i}$ : such an $a$ exists because of the irredundance of the union. For each $b \in A_{S} \backslash A_{S \cup\{u\}}$ there exists $t \in \mathbb{Z} \backslash S \cup\{u\}$ for which $a^{-1} b \in A_{r}$. It follows that $A_{S} \backslash A_{S \cup\{u\}} \subseteq \cup\left(a A_{i}: t \notin S \cup\{u\}\right)$. If $a A_{t} \cap A_{S} \neq \varnothing$, then $a A_{t} \cap A_{S}=g_{t} A_{S \cup\{t\}}$ for some $g_{t} \in A_{S}$. In other words (2.4) is proved.

Now let $h$ be a p-element of $G$. We prove by induction that, for each $m$ satisfying $1 \leq m \leq n$, there is a subset $S$ of $\mathbf{Z}$ of cardinality $m$, for which $A_{S}$ contains $h$. For $m=1$ this is true because $G$ is the union of subgroups $A_{s}(1 \leq s \leq n)$. Suppose that, for some $m$ satisfying $1 \leq m \leq n$, there is a subset $S$ of $\mathbf{Z}$ of cardinality $m$ for which $A_{s}$ contains $h$. By (2.4), $h^{i}(1 \leq i \leq p-1)$ belongs to

$$
\cup\left(g_{t} A_{S \cup(t)}: t \in T\right)
$$

where $T$ is a non-empty subset of the complement of $S$ in $Z$. Now one at least of the cosets $g_{t} A_{S \cup\{t\}}$ is a subgroup. If some $h^{i}(1 \leq i \leq p-1)$ is in this subgroup then so is $h$, and we have completed the inductive step. If not, then the $p-1$ powers of $h$ lie in the union of $|T|-1 \leq n-m-1 \leq n-2<p-1$ of the cosets $g_{t} A_{S \cup(t)}$. One, therefore, contains powers $h^{i}$ and $h^{j}$ for $1 \leq i<j \leq p-1$. It follows that $h^{j-i}$, and therefore $h$, is in $A_{S \cup(t)}$ for some $t \in T$. Since $|S \cup\{t\}|=m+1$, this completes the induction.

Since $D=A_{S}$ for the unique $S$ for which $|S|=n$, we have proved the claim of the lemma.

We will use repeatedly, without explicit mention, that no group is the union of two proper subgroups.

\section{Proof of Theorem 1.2}

We now turn to Theorem 1.2. First note that it is sufficient to prove that the groups of the class $\mathscr{H}_{n}(p)$ are $p$-nilpotent; for Lemma 2.1, and the result of Thompson [5], ensures that a normal $p$-complement is nilpotent. The proof will be by contradiction. Suppose that there is a positive integer $n$ and a prime $p>n$ for which $\mathscr{H}_{n}(p)$ contains 
groups which are not $p$-nilpotent. It follows from Theorem 1.1 that $n \geq 2$ and therefore that $p \geq 3$. Moreover let $n$ be the least such integer, and let $G \in \mathscr{H}_{n}(p)$ be a minimal non-p-nilpotent group. $G$ has an $n$-union $\mathscr{A}$ with the Hughes property for exponent $p$, but no $(n-1)$-union with this property.

For convenience we write $\mathscr{A}=A_{1} \cup A_{2} \cup \ldots \cup A_{n}$ and $D=\cap \mathscr{A}$.

As a consequence of the next result we are able to choose the subgroups $A_{i}(1 \leq i \leq n)$ as maximal subgroups of $G$.

(3.1). $G$ is not the union of $n$ maximal subgroups.

Suppose, on the contrary, that $G$ is the union of $n$ maximal subgroups. Then it is the irredundant union of maximal subgroups $M_{i}(1 \leq i \leq m)$ where $1<m \leq n$. Write $E=$ $M_{1} \cap M_{2} \cap \ldots \cap M_{m}$.

Since $p \geq m$ all $p$-elements of $G$ lie in $E$ by Lemma 2.2 . Let us write $S$ for the subgroup they generate. Now $S$ is of smaller order than $G$, and it is not contained in $\mathscr{A}$ so, by the minimality of $G$ it, and every proper subgroup containing it, has a normal $p$ complement. Moreover $S$ is normal in $G$, and $G / S$ is a $p^{\prime}$-group. But $S \subseteq O_{p^{\prime} p}(G):=R$, so $G / R$ is a $p^{\prime}$-group. Since every proper subgroup of $G$ containing $S$ has a normal $p$ complement, it must be that $R$ is of prime index in $G$.

Let $P$ be a Sylow $p$-subgroup of $G$ chosen so that $P \nsubseteq \mathscr{A}$. Write $T=\mathbf{N}_{G}(P)$. By the Frattini argument* $G=R T$. Therefore $P \subset T$. If $T \neq G$ then it is $p$-nilpotent. However this means that some $p^{\prime}$-element centralises an element outside $\mathscr{A}$, a contradiction to Lemma 2.1. Therefore $T=G$. For the same reason we must have $O_{p^{\prime}}(G)=1$. Hence $P=R$ is maximal in $G$. However, by Lemma $2.2, P \subseteq E$, which is not maximal. This contradicts the fact that $G$ is the union of the subgroups $M_{i}$, completing the proof of (3.1).

It follows now that we may replace the subgroups $A_{i}$ by maximal subgroups containing them, and still have an $n$-union in $G$ with the Hughes property. To avoid introducing new notation we suppose that the subgroups $A_{i}$ are all maximal.

(3.2). If $U$ is an abelian minimal normal subgroup of $G$ with $|U|>n$ then $U \subseteq D$.

Since $A_{i}$ is maximal, and $U$ abelian and minimal normal, either $A_{i} \cap U=1$ or $U \subseteq A_{i}(1 \leq i \leq n)$. Suppose that $U$ is not in $D$. Then $U \cap A_{1}=1$, say.

Now there exists $a \in A_{1}$ which lies in none of the subgroups $A_{i}(2 \leq i \leq n)$, or else $G$ would be in the class $\mathscr{H}_{n-1}(p)$. It follows that, for each $i \in\{2,3, \ldots, n\}$, there is at most one element $u_{i}$ of $U$ for which $a u_{i}$ is in $A_{i}$. Since $|U|>n$ it follows that $a u_{0}$ is not in $\mathscr{A}$ for at least one $u_{0}$ in $U$. This means that $1=\left(a u_{0}\right)^{p}=a^{p} u^{\prime}$ for some $u^{\prime}$ in $U$, which in turn means that $a^{p} \in A_{1} \cap U=1$. Since $a$ is an arbitrary element of $A_{1}$ not in $A_{2} \cup A_{3} \cup \ldots \cup$ $A_{n}$ it follows that $G$ is in $\mathscr{H}_{n-1}(p)$, a contradiction. Hence $U$ is in $D$, as we required.

Let $P$ be a Sylow $p$-subgroup of $G$ chosen so that $P$ is not contained in $\mathscr{A}$, and let $J$ be the subgroup of $P$ generated by the abelian subgroups of $P$ of maximal order. We write $Z$ for the centre of $J$, and $N$ for the normaliser of $Z$ in $G$. By a theorem of Glauberman (Chaper $\mathrm{X}, 9.10$ in [3]), $N$ is not $p$-nilpotent. However $N$ is not in $\mathscr{A}$, since $P$ is not, so the minimality of $n$ and $|G|$ ensures that $N=G$.

\footnotetext{
* Professor Zappa points out (I contributi di Alfredo Capelli alla teoria dei gruppi, Bollettino di Storia delle Scienze Matematiche XI (1991), 25-54) that the priority for this idea belongs to Capelli and not to Frattini.
} 
Now let $M$ be a minimal normal subgroup of $G$ contained in $Z$. Since $|M| \geq p>n$ it follows from Lemma 3 that $M \subseteq D$. Hence, since $|G / M|<|G|$ and since $G / M$ satisfies the hypotheses of the theorem, $G / M$ is $p$-nilpotent. Write $W$ for the subgroup of $G$ defined by $W / M=O_{p^{\prime}}(G / M)$, and let $x$ be an element of $G$ outside both $W$ and $\mathscr{A}$. Then the subgroup $W\langle x\rangle$ is not in $\mathscr{A}$. If it were a proper subgroup of $G$ then it would be $p$ nilpotent. In particular $W$ would be $p$-nilpotent. However this would mean that $O_{p^{\prime}}(W)=O_{p^{\prime}}(G)$ with $G / O_{p^{\prime}}(G)$ a $p$-group, contradicting the non-p-nilpotence of $G$. Hence $G=W\langle x\rangle$.

Now $G / M$ is in $\mathscr{H}_{n}(p)$ so, by Lemma $2.1, x$ acts fixed point freely on $W / M$. Therefore, by Thompson [5], $W / M$ is nilpotent. In particular $G$ is soluble. We now show that $G$ is monolithic. Let $X$ be a minimal normal subgroup other than $M$. If it is a $p$-group it is in $D$ by (3.2). If it is a $p^{\prime}$-group then $x$ acts without fixed points on it, and it follows that $|X|>p \geq n$. Hence again, by (3.2), $X \subseteq D$. However in either case this means that $G / X$ is $p$-nilpotent by induction, and so $G$ is a subdirect product of $G / M$ and $G / X$ and therefore $p$-nilpotent, contradiction. Hence $M$ is the unique minimal normal subgroup of $G$.

It follows that $M=F(G)$. Let $Y / M$ be a minimal normal subgroup of $G / M$, so that $Y / M$ is a $p^{\prime}$-group. Since $C_{Y / M}(x)=1$, it follows from a theorem of Higman (Chapter IX, 1.10 in [3]) that the minimum polynomial of $x$ on $M$ is $(x-1)^{p}$. Therefore there is an element of order $p^{2}$ outside $\mathscr{A}$. This final contradiction concludes the proof of Theorem 1.2.

\section{Proof of Theorem 1.3}

The crux of the proof of Theorem 1.3 is in the following two results. After stating them, and before proving them, we show how they may be used to give a short proof of Theorem 1.3.

Lemma 4.1. Let $G$ be a finite group containing an $n$-union $\mathscr{A}$ with the Hughes property for exponent $p$, but no $(n-1)$-union with the same property. Then $O_{q}(G) \subseteq \cap \mathscr{A}$ for all primes $q$ greater than or equal to $n$, but different from $p$.

Lemma 4.2. Let $G$ be a group in the class $\mathscr{H}_{n}(p)$, and suppose that $q$ is a prime different from $p$, and greater than or equal to $n$. Then $G$ has a unique Sylow q-subgroup.

Proof of Theorem 1.3. Let $q \in \pi$. By (4.2) there is a unique Sylow $q$-subgroup $S_{q}$ of $G$. Therefore $S_{q}$ is normal in $G$, and the subgroup $H$ generated by all $S_{q}(q \in \pi)$ is nilpotent and normal in $G$. Of course $G / H$ is a $\pi^{\prime}$-group. This completes the proof of Theorem 1.3.

Proof of (4.1). Let $D=\cap \mathscr{A}$. Now suppose that $N$ is a normal $q$-subgroup of $G$ for some prime $q$ greater than or equal to $n$, but not equal to $p$. Moreover, in order to obtain an eventual contradiction, suppose that $N \nsubseteq D$. Let $h \in N \backslash D$. We write $\mathscr{A}=$ $A_{1} \cup A_{2} \cup \ldots \cup A_{n}$ with the notation chosen so that $h \in A_{i}$ for $1 \leq i \leq m$ and $h \notin A_{i}$ for $m+1 \leq i \leq n$. Note that $1 \leq m<n$. Choose $a \in A_{n}$ not belonging to any of the other $A_{i}$ : such an element exists because, by hypothesis, $A_{n}$ cannot be omitted from the union. Then, for $1 \leq j \leq q-1, a h^{j} \notin A_{1} \cup A_{2} \cup \ldots \cup A_{m} \cup A_{n}$. 
Now for each $i \in\{m+1, \ldots, n-1\}$, if any, there is at most one $j \in\{1,2, \ldots, q-1\}$ such that $a h^{j} \in A_{i}$; or else $h$ would be in one of these $A_{i}$. However $q-1 \geq n-1>n-m-1$ and so, for at least one $k \in\{1,2, \ldots, q-1\}, a h^{k} \notin \mathscr{A}$. Hence there exists an element $h_{0} \in N$ for which $a h_{0} \notin \mathscr{A}$; in particular it has order $p$. Therefore

$$
1=\left(a h_{0}\right)^{p}=a^{p} h_{0}^{\alpha^{p-1}} h_{0}^{\alpha^{p-2}} \ldots h_{0}^{\alpha} h_{0}
$$

We show that $v:=h_{0}^{\alpha^{p-1}} h_{0}^{\alpha^{p-2}} \ldots h_{0}^{\alpha} h_{0}=1$. Suppose not, and let $N_{i}$ be the first term of the lower central series of $N$ not containing $v$. Note that $i \geq 2$. Now $a h_{0}$ acts by conjugation on the factor group $N_{i-1} / N_{i}$ and, in this action, $N_{i} v$ is a fixed point because

$$
v^{a h_{0}} \equiv v^{a}=h_{0}^{\alpha^{p}} v h_{0}^{-1}=h_{0}^{v^{-1}} v h_{0}^{-1} \equiv v \bmod \left(N_{i}\right)
$$

However $a h_{0}$ has no non-trivial fixed points in $N$, by Lemma 2.1 , so none in $N / N_{i}$. It follows that $v \in N_{i}$, a contradiction to the choice of $i$. Therefore $v=1$ as claimed.

From this it follows that $a^{p}=1$. Since $a$ is an arbitrary element of $A_{n} \backslash A_{1} \cup A_{2}$ $\cup A_{3} \ldots \cup A_{n-1}$ this would mean that $A_{n}$ could be omitted from the union, contrary to hypothesis.

This completes the proof of (4.1).

Proof of (4.2). We suppose not, and that $n$ is the least integer for which a counter-example $G$ exists. By Theorem 1.1 and $[1], n \geq 3$. We assume, moreover, that $|G|$ is as small as possible. We write $\mathscr{A}=A_{1} \cup A_{2} \cup \cdots \cup A_{n}$ for an $n$-union in $G$ with the Hughes property, and we write $D=\cap \mathscr{A}$. There is more than one Sylow $q$-subgroup of $G$.

First we prove

(4.3). $\quad O_{q}(G)=1$.

For, it follows from (4.1) that $O_{q}(G) \subseteq D$. Now the factor group $G / O_{q}(G)$ also satisfies the hypotheses of (4.2), and it has more than one Sylow $q$-subgroup, so the minimality of $G$ means that $O_{q}(G)=1$.

(4.4). If $H$ is a proper subgroup of $G$ which contains more than one Sylow q-subgroup of $G$, then $H \subseteq \mathscr{A}$.

For, otherwise, $H$ and the $m$-union $H \cap \mathscr{A}(m \leq n)$ satisfy the hypotheses of (4.2). But then either $m<n$, or $m=n$ and $|H|<|G|$ so, by the minimal choice of $n$ and $G, H$ has a unique Sylow $q$-subgroup, a contradiction.

(4.5). The subgroups $A_{i}$ may be chosen maximal.

If not, then $G$ is the union of $n$ maximal subgroups containing the $A_{i}$, and therefore the irredundant union of at least three of them. As in the proof of (3.1), the subgroup $S$ generated by the $q$-elements of $G$ is proper, but not maximal, in $G$. Let $x \in G \backslash \mathscr{A}$. Then 
$S\langle x\rangle=G$ by (4.4). But $S\langle x\rangle \neq G$ because $S$ is not maximal in $G$. This completes the proof of (4.5), and we continue with the proof of (4.2).

(4.6). $\Phi(G)=1$.

By (4.5) the subgroups $A_{i}(1 \leq i \leq n)$ are all maximal. Therefore $\Phi(G) \subseteq D$. Hence, unless $\Phi(G)=1, G / \Phi(G)$ satisfies the hypotheses of (4.2) and has order smaller than that of $G$, so that $S \Phi(G) / \Phi(G)$ is normal in $G / \Phi(G)$. It follows that $G=\mathbf{N}_{G}(S) \Phi(G)=\mathbf{N}_{G}(S)$, a contradiction and therefore $\Phi(G)=1$.

By Lemma 2.2, no $q$-group can be the union of fewer than $q+1$ proper subgroups. Hence every Sylow $q$-subgroup of $G$ lies in some $A_{i}$. By Sylow's theorem there are at least $q+1$ Sylow $q$-subgroups of $G$. It follows from the pigeon-hole principle that $A_{1}$, say, contains at least two of these Sylow subgroups. We write $S_{i}$ for the subgroup of $A_{i}$ generated by all its $q$-elements $(1 \leq i \leq n)$. Without loss of generality we may suppose that $S_{1}$ is of maximal order among these subgroups $S_{i}$; and that $S_{2}$ is of maximal order among the subgroups $S_{i}(2 \leq i \leq n)$.

(4.7). $S_{i}$ is a Sylow subgroup of $G$ for $2 \leq i \leq n$.

It will suffice to show that $S_{2}$ is a Sylow subgroup of $G$. We suppose that it is not, in order to obtain a contradiction. We show first that

(4.8). $S_{2}$ is not normal in $G$.

Otherwise $S_{2}\langle x\rangle=G$ by (4.4), so $S_{2}$ is maximal, and therefore $A_{2}=S_{2}$. Also it follows that $A_{2}$ contains all $q$-elements of $G$, whence $A_{2}=S_{2}=S_{1} \subseteq A_{1}$. However this contradicts the minimal choice of $n$, so $S_{2}$ is not normal in $G$.

Another consequence of (4.4) is that, for all $g \in G, A_{2}^{g} \subseteq \mathscr{A}$. In fact we show now that

(4.9). for all $g \in G$, there exists a jor which $A_{2}^{g}=A_{j}$.

Because $A_{2}$ is maximal in $G$, the result is true if $A_{2}^{g} \subseteq A_{j}$ for some $j$. Hence, if the statement of (4.9) is false, then $A_{2}^{g}$ is the union of at least three of its intersections with the subgroups $A_{i}(1 \leq i \leq n)$. By Lemma 2.2 therefore, there are integers $j, k$ satisfying $2 \leq j<k \leq n$ for which $S_{2}^{g} \subseteq S_{j} \cap S_{k}$, whence $S_{2}^{g}=S_{j}=S_{k}$. However $A_{j}$ and $A_{k}$ together generate $G$ by (4.5). Hence $S_{2}^{g} \unlhd G$, and therefore $S_{2} \unlhd G$, a contradiction to (4.8).

Next we show that

(4.10). $\left|G: A_{2}\right|=q=n$.

Since $A_{2}$ is maximal, and not normal in $G$, it is self-normalising. It follows that $\left|G: A_{2}\right| \leq n$ since, by (4.9), there are at most $n$ distinct conjugates of $A_{2}$. However, if either $n<q$ or $\left|G: A_{2}\right|<n$ then $A_{2}$ contains every $q$-element of $G$, contradicting the non-normality of $S_{2}$. This proves (4.10).

It now follows that the conjugates of $A_{2}$ are precisely the subgroups $A_{i}(1 \leq i \leq n)$. The orbits of these subgroups under conjugation by an element $x \notin \mathscr{A}$ have length either 1 or $p$. Since the sum of the orbit lengths is $q$, there must be at least one fixed point for $x$, that is a subgroup $A_{j}$ normalised by $x$. However this says that $A_{j} \unlhd G$, whence all the subgroups $A_{i}$ are equal, being conjugate, a contradiction. 
This completes the proof of (4.7).

(4.11). $A_{1}=S_{1}$ and this subgroup is normal in $G$.

By (4.4), $A_{1}^{g} \subseteq \mathscr{A}$ for all $g \in G$. If $A_{1}^{g} \neq A_{1}$ then Lemma 2.2 yields that $S_{1}^{g} \subseteq A_{i}$ for at least one $i \geq 2$, contradicting (4.7) since $S_{1}$ is not a Sylow subgroup of $G$. It follows that $A_{1} \unlhd G$, whence $S_{1} \unlhd G$. As in the proof of (4.8), we get that $A_{1}=S_{1}$ as required.

Our aim now is to show that

(4.12). $A_{1}$ has a normal $q$-complement $K$.

By (4.7) there is a Sylow q-subgroup $S$ of $G$ not in any of $A_{i}(2 \leq i \leq n)$. Of course $S$ is in $A_{1}$. Since $A_{1} \unlhd G, G=A_{1} \mathbf{N}_{G}(S)$ by the Frattini argument and therefore, in particular, $\mathbf{N}_{G}(S) \nsubseteq A_{1}$.

Now let $Z$ be a characteristic subgroup of $S$. Write $N=\mathbf{N}_{G}(Z)$. Note that $N \supseteq \mathbf{N}_{G}(S)$ hence $N \nsubseteq A_{1}$. In fact $N \nsubseteq \mathscr{A}$ because otherwise Lemma 2.2 would require that $S$ belong to some $A_{i}(i>1)$. By (4.3) $N \neq G$.

Because $N \nsubseteq \mathscr{A}, N \in \mathscr{H}_{r}(p)$ for some $r \in\{1,2, \ldots, n\}$, where $N \cap \mathscr{A}$ is an $r$-union with the Hughes property for some $r \leq n$. Choose $r$ to be as small as possible. Since $G$ is minimal, $S$ is normal in $N$. If $r>1$ then, by (4.1), $S \subseteq A_{i}$ for some $i>1$. This contradicts the choice of $S$. Hence $r=1$. This must mean that every element of $N$ outside $N \cap A_{1}$ has order $p$, because there are $q$-elements in $N \backslash A_{i}$ for every $i>1$. Theorem 1.1 then yields that $N \cap A_{1}$ is nilpotent. Therefore $\mathbf{N}_{A_{1}}(Z)$ is nilpotent.

Now we may choose $Z$ to be the centre of the subgroup of $S$ generated by the abelian subgroups of $S$ of maximal order. Since $3 \leq n \leq q$, Glauberman's Theorem (Chapter X, Theorem 9.10 in [3]) says that $A_{1}$ has a normal $q$-complement, $K$ say, as required by (4.12).

(4.13). The q-complement $K$ is a Sylow subgroup of $A_{1}$, and $G=K S\langle x\rangle$ for some $x \in \mathbf{N}_{G}(S) \backslash \mathscr{A}$.

Let $R$ be a Sylow subgroup of $K$. By the Frattini argument, $G=\mathbf{N}_{G}(R) K$. In particular $L:=\mathbf{N}_{G}(R) \nsubseteq A_{1}$ and $L$ contains a Sylow $q$-subgroup of $G$. Hence some conjugate of $L$ contains $S$. Therefore, with the choice of a suitable conjugate of $R$ originally, we may suppose that $L$ contains $S$. Hence $L \nsubseteq \mathscr{A}$ or else, by Lemma $2.2, S$ would be contained in some $A_{i}$ for $i>1$.

Now $S$ does not centralise $K$ since it is not normal in $G$. Therefore some choice $R$ is not centralised by $S$. For such a choice it follows that $L=G$, since otherwise $L$ would be a smaller counterexample than $G$ to the conclusion of (4.2).

We have seen that $\mathbf{N}_{G}(S) \nsubseteq A_{1}$, and hence $\mathbf{N}_{G}(S) \nsubseteq \mathscr{A}$, or else $S$ would be in some $A_{i}$ with $i>1$. Hence there is an element $x \notin \mathscr{A}$ normalising $S$. Now $G=R S\langle x\rangle$ satisfies the hypotheses of (4.2), and is a counterexample to its conclusion. Since $G$ is minimal therefore, $G=R S\langle x\rangle$. It follows that $R=K$, so (4.13) is proved.

Since $\Phi(G)=1, K$ is elementary abelian. It is not centralised by $S$ since $S$ is not normal in $G$. By Fitting's Lemma therefore, there is a minimal normal subgroup $M$ of $G$ contained in $K$, which is not centralised by $S$. Then $M S\langle x\rangle$ is a subgroup satisfying the 
conditions of (4.2) and therefore, $G=M S\langle x\rangle$. Moreover $M$ is (irreducible and) faithful for the action of $S\langle x\rangle$, since $O_{q}(G)=1$ by (4.3).

From this, and the minimality of $G$, it follows that $S M / M$ is a chief factor of $G$. Higman's theorem (Chapter IX, 1.10 in [3]) shows that the minimum polynomial of $x$ on $M$, is $x^{p}-1$. Therefore $M$ is a $p$-group since, by Lemma $2.1, x$ centralises no $p^{\prime}$-elements. We deduce that, for some element $m$ of $M, y=x m$ is of order $p^{2}$.

Note that $C_{G}(y)$ is a $p$-group, otherwise $x$ would have fixed points in $S$. Choose $s \in S$, $s \neq 1$. Consider the set of conjugates $\left\{y^{s j}: 0 \leq j \leq q-1\right\}$. This set has cardinality $q$. These elements are contained in $\cup\left(A_{i}: 2 \leq i \leq n\right)$ and hence one of the subgroups $A_{i}(2 \leq i \leq n)$ contains two of them. However two of these elements together generate $G$ giving a final contradiction, concluding the proof of (4.2), and with it the proof of Theorem 1.3.

Acknowledgement. The first author acknowledges with gratitude financial support from C.N.R., and the hospitality of the Department 'Ulisse Dini' of the University of Florence where this work was carried out.

\section{REFERENCES}

1. R. A. Bryce, On a Theorem of Hughes and Thompson, Bull. Austral. Math. Soc. 50 (1994), 41-48.

2. D. R. Hughes and J. G. Thompson, The $H_{p}$-problem and the structure of $H_{p}$-groups, Pacific J. Math. 9 (1959), 1097-1101.

3. B. Huppert, and N. Blackburn, Finite groups (Springer-Verlag, Berlin-Heidelberg-New York, 1982).

4. Otto H. KeGEL, Die Nilpotenz der $H_{p}$-gruppen, Math. $Z$. 75 (1961), 373-376.

5. J. G. Thompson, Finite groups with fixed point free automorphisms of prime order, Proc. Nat. Acad. Sci. U.S.A. 45 (1959), 578-581.

R. A. BRYCE

School of Mathematical Sciences

The Australian National University

Canberra, A.C.T. 0200

Australia
V. Fedri and L. Serena

Dipartimento di Matematica

viale Morgagni, 67/A

50134 FIRENZE

ITALY 